\title{
Glyoxylate Dehydrogenase (Acylating)
}

National Cancer Institute

\section{Source}

National Cancer Institute. Glyoxylate Dehydrogenase (Acylating). NCI Thesaurus. Code C86977.

An oxidoreductase that catalyzes the conversion of Coenzyme A to oxalyl-Coenzyme A and the reduction of NADP+ to form NADPH through the cleavage of a glyoxylate molecule. This enzyme is involved in the biosynthesis of carbohydrate from fatty acids or other precursors. 